\title{
RECOMMENDATION SYSTEM BASED ON NEURAL NETWORK FOR PREDICTION OF EMERGENCY SituATIONS
}

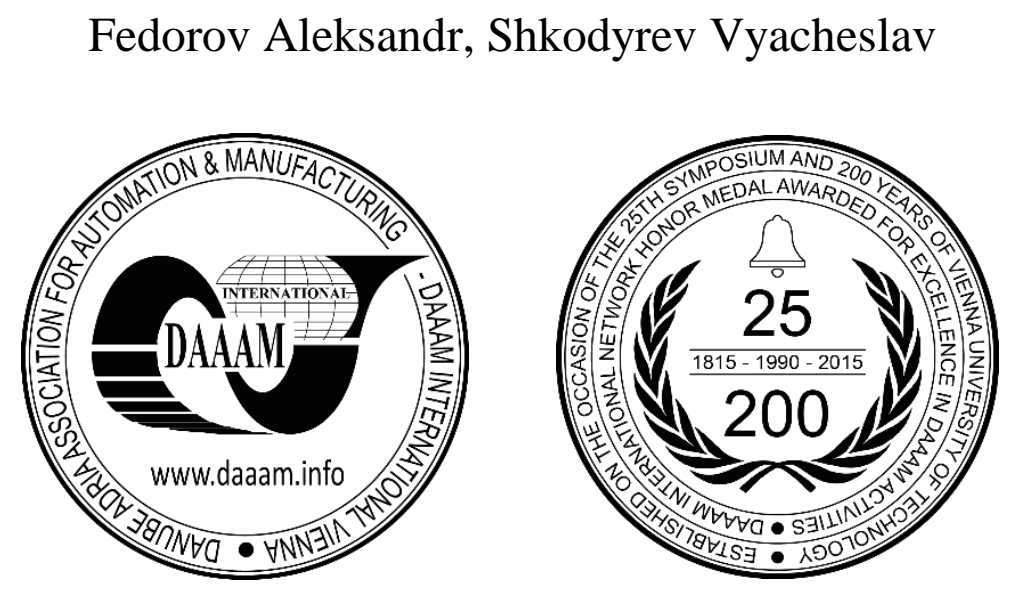

This Publication has to be referred as: Fedorov, A[leksandr] \& Shkodyrev, V[iacheslav] (2018). Recommendation System based on Neural Network for Prediction of Emergency Situations, Proceedings of the 29th DAAAM International Symposium, pp.0133-0136, B. Katalinic (Ed.), Published by DAAAM International, ISBN 978-3-902734-20-4, ISSN 1726-9679, Vienna, Austria

DOI: $10.2507 / 29$ th.daaam.proceedings.018

\begin{abstract}
In this work, we investigate a new approach of proactive recommendation systems proposed by authors in the field of predicting emergency situations on road and manufacturing. A new class of algorithms and methods of preventive control for poor formalized, fast-acting situations includes approach of the neural-fuzzy controller, knowledge base and deep learning, which helped to get precision near 0.69 with recall 0.80 in the task of detecting emergencies from dashcam videos outperforming other baselines without the combination of such methods. Developed hybrid recommendation system that can assist in the selection of an optimal sequence of actions to prevent the emergency. The main contribution of authors in the applying of methods from formal models of fuzzy logic, neural networks, and expert systems, which shows great results in preventive forecasting of any type of emergency situations.
\end{abstract}

Keywords: deep learning; neural networks; recommendation systems; machine vision

\section{Introduction}

In this paper, we propose one of the possible solutions to unforeseen accidents in transport systems - situational control. The aim of the study is to develop and study a system for recognizing and predicting poorly structured, rapidly evolving situations in the face of uncertainty based on a hybrid neural network model using a deep learning algorithm for driving a vehicle.

The object of the study is poorly formalized scenarios of dynamic scenes with rapidly changing parameters in conditions of uncertainty when driving. The ability to prevent accidents is an extremely difficult task, since accidents are very diverse and, as a rule, they happen suddenly.

Drivers learn from experience to pay attention to implicit signals, including the semantics of the scene, the appearance of the object and movement. This paper proposes a situational control based on a recurrent neural network (RNN) to predict accidents before they occur. 
The system consists of such components as:

- NNFLC - neuro-fuzzy controller for outputting human-readable commands or descriptions of the current situation

- $\quad$ RNN for predicting an emergency is used RNN with Long Short Term Term (LSTM) cells to simulate the longterm dependencies of all signals and predict accidents [1], [2].

- Exponential-loss: by analogy of predicting driver maneuvers, the exponential descending function is used as a loss function for positive examples [3], [4].

For effective forecasting, the deep learning method is used. All these components together provide prediction of accidents based on low-cost sensor based on machine vision [5], [6]. The software module developed as a result of the work includes a full-fledged application and adaptation for the Raspberry Pi 3B microcomputer, and the standard Tensorflow model for pattern recognition "inception graph" was supplemented with a trained recurrent neural network based on 1730 videos of the VSLab from video recorders in Taiwan. An example of the program is shown in Fig. 1-3.

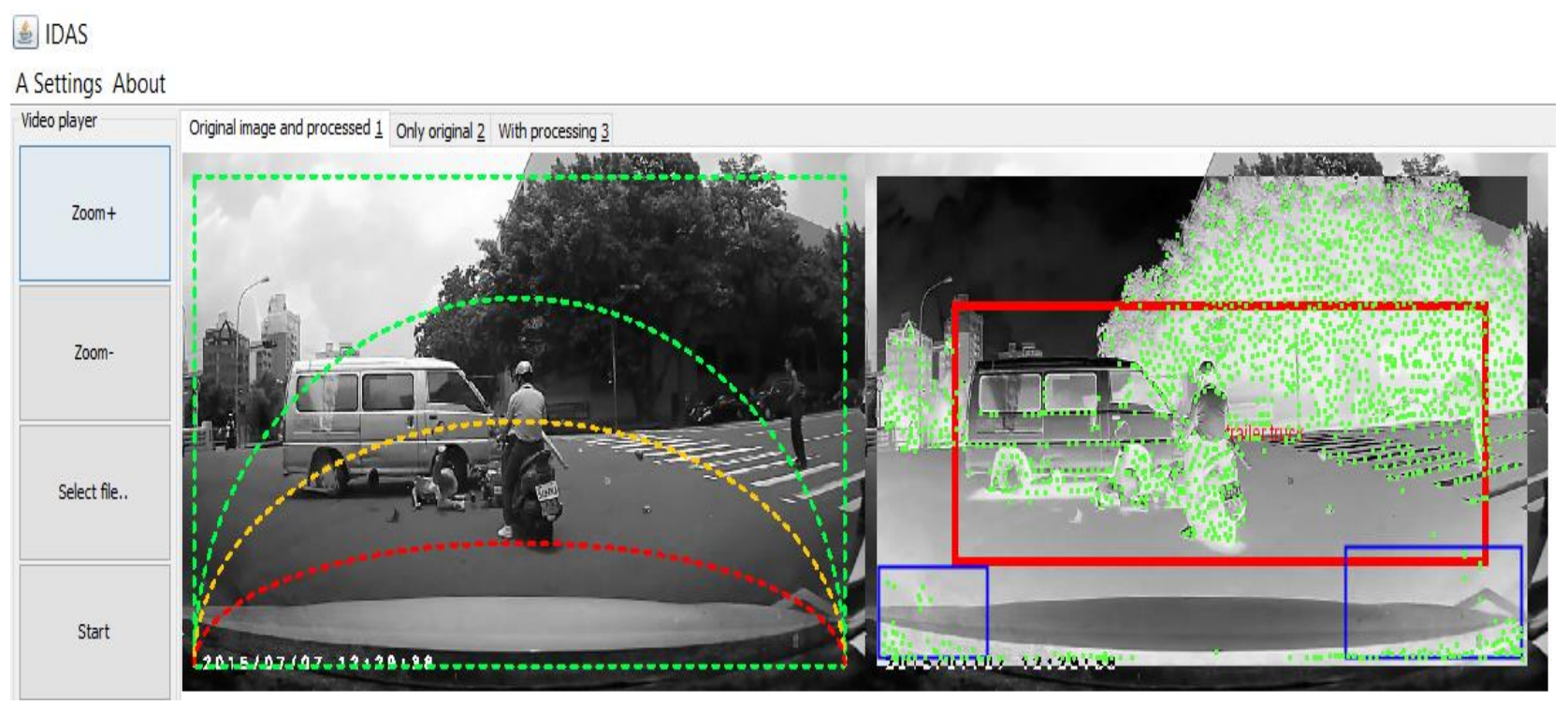

Fig. 1. The principle of operation of the algorithm over time - the system detects an emergency and highlights it accordingly.

\section{包 IDAS}

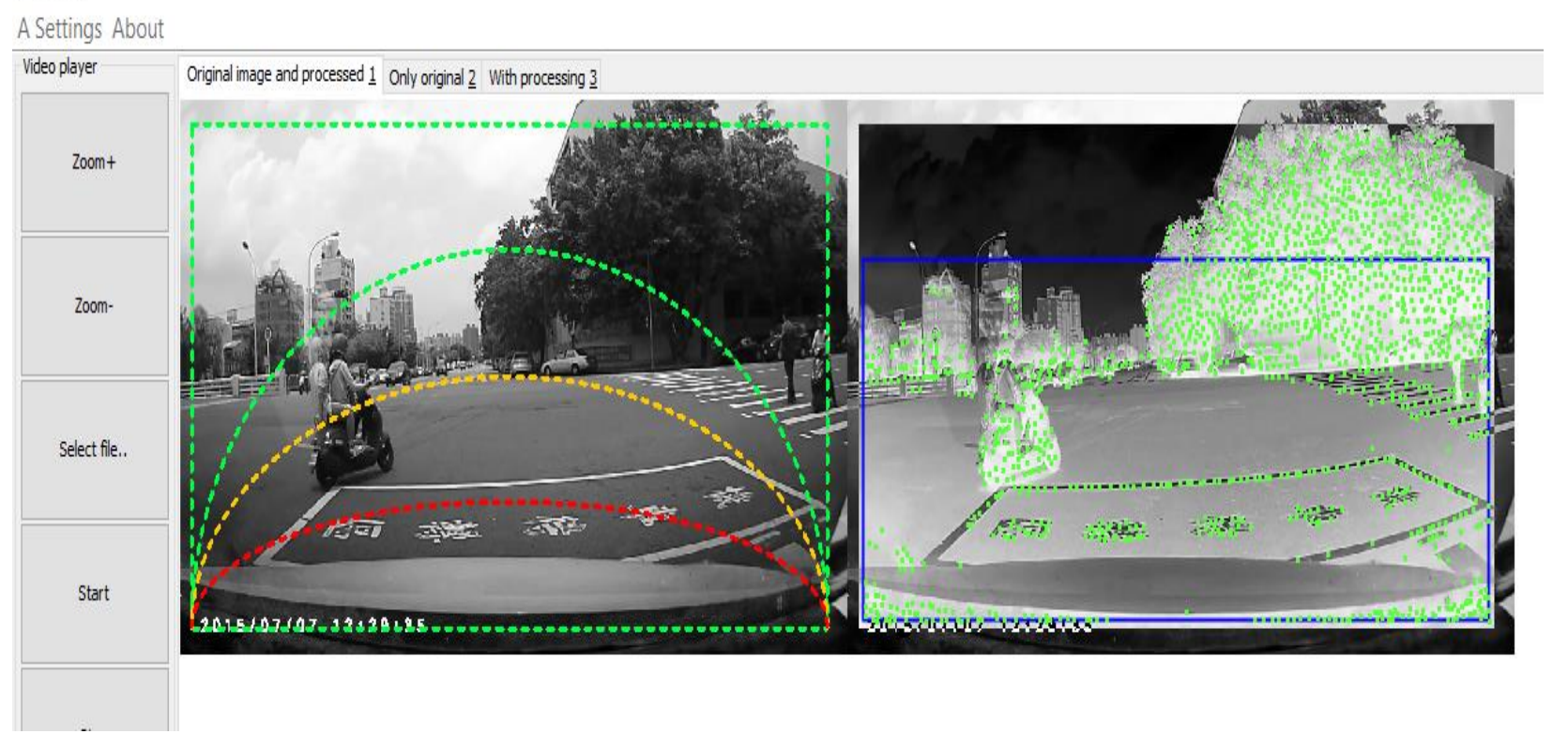

Fig. 2. The principle of operation of the algorithm over time - the system did not detect an emergency. 


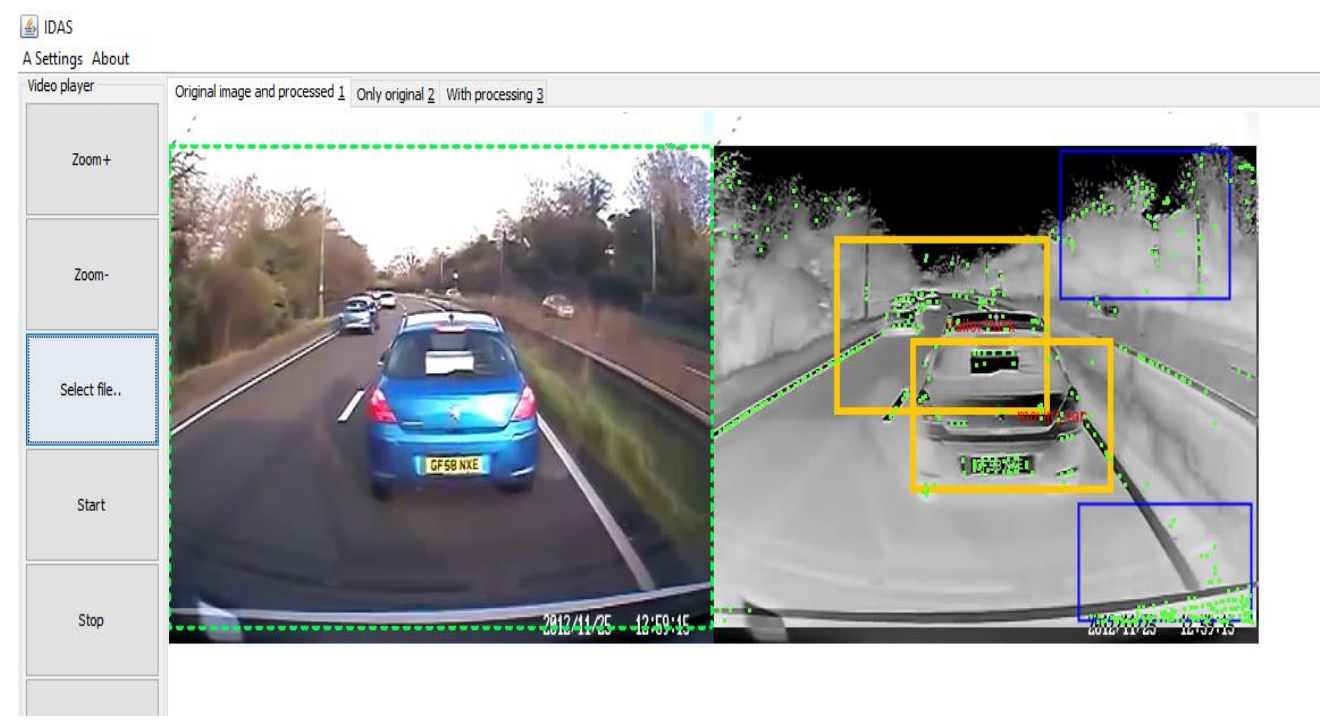

Fig. 3. The principle of the algorithm operation over time - first, the presence of moving objects is determined and the area around them is selected using the SURF and DBSCAN algorithm, then the RNN determines what this object belongs to and the type of emergency situation is determined using NNFLC on screen.

\section{Training and test examples}

Training and test sample for training a hybrid neural network in transport systems consist of large dataset of video samples. The training and testing of the hybrid network model was carried out based on records from DVRs from VSLab in Taiwan; a total of 1,730 records were provided, 100 frames each, with a resolution of 720p [5]. This set of videos and served as data for testing the model.

The distribution of accidents on video is as follows $-42.6 \%$ of the motorcycle collides with the car, $19.7 \%$ of the car collides with the car, $15.6 \%$ of the motorcycle collides with the car and $20 \%$ other types of accidents.

Below are the graphs obtained as a result of training the model and test run based on the test sample.
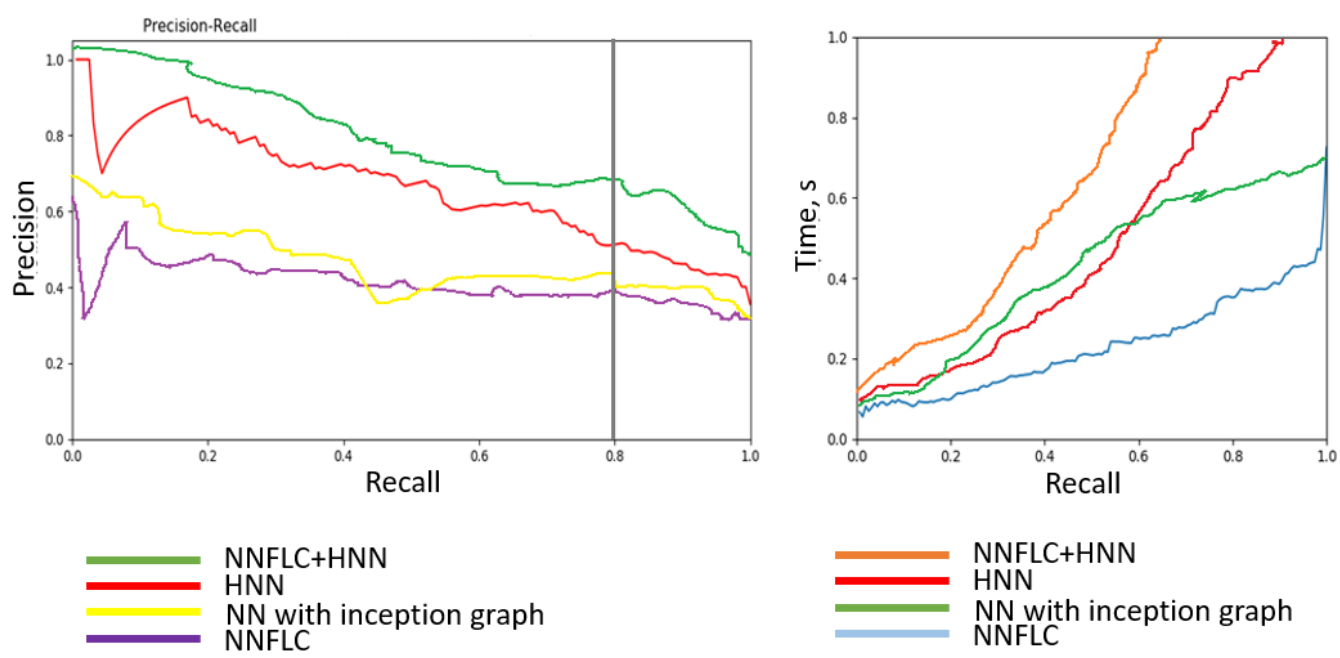

Fig. 4. The first graph shows the dependence of Precision vs. Recall (PR). The second graph shows the average time until the time of the accident Time-to-Accident for different Recall (ToAR).

As can be seen from the graph, the method including NNFLS and HNN is able to predict an accident in average for $1.4 \mathrm{~s}$ before it occurs with a precision of 0.76 and $80 \%$ recall (the ratio of correctly guessed objects of a class to all members of this class).

\section{Prototype}

It is possible to control the situation under the control system. If you are not planning on a system of all system parameters or boundary values are unknown. It was 1.730 . hybrid neural network was developed. 
But it also requires further improvement. This is a video camera that can be used to predict accidents. During the study of RNN model (recurrent neural network) for this paperwork, the following parameters were applied and derived empirically:

1. The parameters of the learning algorithm:

a. Learning rate (learning rate): 0.0001

b. number of "epochs" (cycles): 60

c. Batch-size for batch normalization: 10

2. Network settings:

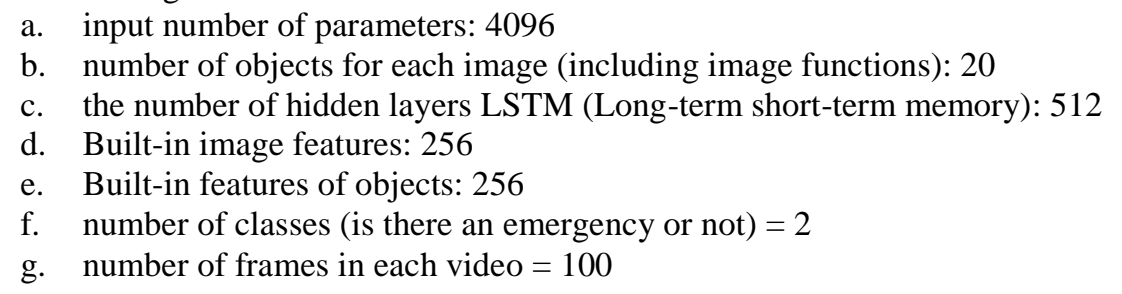

Based on these parameters, as well as 1730 videos, training and testing of the neural network were conducted. The learning process on a PC with Core i7 $2.8 \mathrm{GHz}$ and $30 \mathrm{~GB}$ of RAM took 8 hours. The hybrid neural network in combination with NNFLC allows defining an emergency situation in 1.8 seconds with $80 \%$ recall and $68.9 \%$ precision. Average precision is $76.6 \%$. The average training time of the model is 8 hours. Experimental conditions:

- $\quad$ PC with a Core i7 processor and $16 \mathrm{~Gb}$ Ram

- Programming language - Java, Python

- Libraries for implementing NN - neuroph, jfuzzylite, pandas, numpy, tensorflow, kitty, apache common math.

These results suggest that the proposed model has the best results among all that allow you to define an emergency.

\section{Conclusion}

The developed hybrid neural structure allows solving vehicle control problems under uncertainty conditions with the possibility of retraining while driving. Mathematical software and a program library of a recommendation system based on a knowledge base of scenario models for operational forecast of situation development scenarios and decision rules can be used in systems that contain a large number of unknown components under conditions of uncertainty, when there cannot be all system parameters or boundary values are unknown. As part of the study, a prototype was developed for reading a video stream and processing data on a Raspberry Pi 3 mobile computer, a data set for training a recurrent neural network consisting of 1,730 videos was developed, a traffic situation monitoring system based on a hybrid neural network was developed. The developed model demonstrated acceptable results on test data, but requires more data and possible further improvement. This paper shows the ability to predict accidents on the roads with the help of simple equipment based on data from a video camera, which can help reduce traffic accidents.

\section{References}

[1] Abdelhadi A. \& Guy P. (2017), A Long Short-Term Memory Recurrent Neural Network Framework for Network Traffic Matrix Prediction, Available from: https://arxiv.org/pdf/1705.05690.pdf

Accessed: 2018-01-13

[2] Francisco J. O.\& Daniel R. (2016). Deep Convolutional and LSTM Recurrent Neural Networks for Multimodal Wearable Activity Recognition, Sensors 2016, 16, 115.

[3] Marchi E. et al. (2014) Multi-resolution linear prediction based features for audio onset detection with bidirectional LSTM neural networks. Acoustics, Speech and Signal Processing (ICASSP), 2014 IEEE International Conference on. - IEEE, pp.2164-2168.

[4] Assaad M.; Boné R. \& Cardot H. (2008) A new boosting algorithm for improved time-series forecasting with recurrent neural networks. Information Fusion. T. 9. №. 1. pp. 41-55.

[5] Fu-Hsiang C.; Yu-Ting C.; Yu X. \& Min S. (2016) Anticipating Accidents in Dashcam Videos, ACCV. Available from: https://yuxng.github.io/chan_accv16.pdf Accessed: 2017-02-01

[6] A. Fedorov; S. Zobnin; V. Shkodyrev; E. Goloschchapov \& V. Potekhin. (2015) Fuzzy decision support in distributed v2v systems. Annals of DAAAM \& Proceedings.Vol. 26. Is. 1. pp. 0097-0103.

[7] Minjia Z.; Samyam R.; Wenhan W. \& Yuxiong H. (2018) Microsoft AI and Research DeepCPU: Serving RNNbased Deep Learning Models 10x Faster. Proceedings of the 2018 USENIX Annual Technical Conference (USENIX ATC'18).

[8] Shuo W. at all. ( 2018) C-LSTM: Enabling Efficient LSTM using Structured Compression Techniques on FPGAs, Available from: https://arxiv.org/abs/1803.06305

Accessed: 2017-12-01 